\title{
Trustworthy Inter-connected Cyber-Physical Systems ${ }^{\star}$
}

\author{
Chris Hankin ${ }^{1}$ (D) and Martín Barrère ${ }^{2}$ (B) \\ 1 Institute for Security Science and Technology, Imperial College London \\ c.hankin@imperial.ac.uk \\ https://www.imperial.ac.uk/people/c.hankin \\ 2 Institute for Security Science and Technology, Imperial College London \\ m. barrere@imperial.ac.uk \\ https://www.imperial.ac.uk/people/m.barrere
}

\begin{abstract}
In this paper we identify some of the particular challenges that are encountered when trying to secure cyber-physical systems. We describe three of our current activities: the architecture of a system for monitoring cyber-physical systems; a new approach to modelling dependencies in such systems which leads to a measurement of the security of the system - interpreted as the least effort that an attacker has to expend to compromise the operation; and an approach to optimising the diversity of products used in a system with a view to slowing the propagation of malware. We conclude by discussing how these different threads of work contribute to meeting the challenges and identify possible avenues for future development, as well as providing some pointers to other work.
\end{abstract}

Keywords: Cyber-Physical Systems · Cyber Security · Critical Infrastructure

\section{Introduction}

There are various estimates of the numbers of devices connected to the Internet but it is likely to be about 30 billion this year with a few thousand additional IoT devices being added every minute. Some companies estimate that there will be a few hundred billion by 2030. Given that each of us can only manage a small number of IT devices, it is inevitable that many of those devices will be deployed in cyber-physical systems (CPS) - domestic heating, lighting and environmental controls, manufacturing processes and critical infrastructures. The rapid pace of digitalisation of these functions and services will pose many new opportunities but also new threats. One source of the new threats is the increasing inter-connectedness of such systems and a greater reliance on computers and communications which in turn rely on a resilient power distribution network. The

\footnotetext{
* Partially supported by EPSRC award EP/R022844/1 (RITICS) and EU Horizon 2020 grant 739551 (KIOS Centre of Excellence).
}

Author's version. Presented at CRITIS 2020. The final authenticated version is available online at https://doi.org/10.1007/978-3-030-58295-1_1. 
rapid growth of numbers of inter-connected devices has out-stripped the ability of legislators to keep up; safety is a major attribute of many cyber-physical systems and it is imperative that these systems can be shown to be trustworthy.

Trustworthy, inter-connected cyber-physical systems are the focus of RITICS, the UK Research Institute in Trustworthy Inter-connected Cyber-physical Systems $^{3}$. The institute is addressing the following key questions:

Q1. Do we understand the harm that cyber threats pose to the provision of critical systems?

Q2. Can we confidently articulate these threats as risk to delivery of critical systems at a business and national level?

Q3. Are there novel effective and efficient interventions for businesses or governments to reduce the risks to critical systems?

Q4. How can we best understand and compare both the effectiveness and costs of potential interventions? These might include technical interventions such as altering system architecture, through to policy interventions by governments and regulators.

Q5. How can we best detect intrusion in critical systems, including embedded and bespoke systems, and how should incident response differ to established practices for enterprise IT? The network traffic in an Industrial Control System (ICS) network is much more predictable than in the Internet, so Intrusion Detection Systems (IDS) can be very effective. Intrusion Prevention Systems (IPS) block unexpected traffic and could be considered to be more risky in an ICS environment - potentially blocking important emergency traffic.

Q6. What are the obstacles to (perceived) best practice being applied to critical systems?

There are many aspects to trustworthiness and our main focus is on cyber security - the system should be protected from interference by malicious attackers. Other aspects include safety, reliability and correctness - where the latter is tested against some formally specified requirements. These different dimensions are clearly inter-related since it is unlikely that an insecure system could be safe

$\overline{3}$ See https://ritics.org.

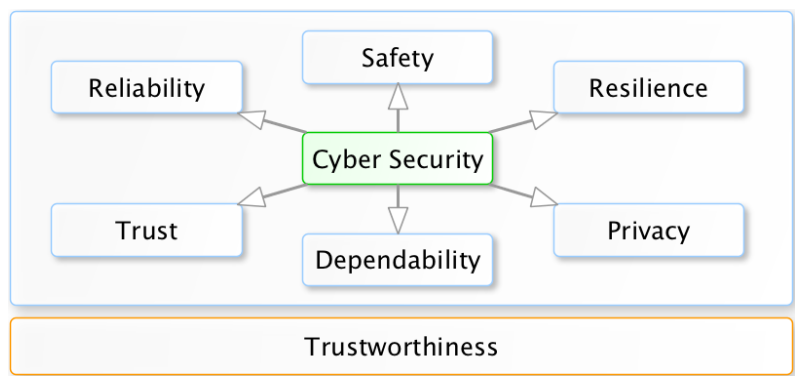

Fig. 1. The role of cyber security for trustworthy inter-connected cyber-physical systems 
or reliable ${ }^{4}$. As illustrated in Fig. 1, our vision is that trustworthiness builds upon a number of fundamental disciplines where cyber security plays a vital role and has an impact on all of them.

\section{Challenges}

There are a number of obstacles and challenges to achieving trustworthiness which include:

C1. Many cyber security specialists start their training as computer scientists and this often leaves them poorly equipped to reason about physical phenomena. It also raises the issue that good solutions for the cyber security of IT systems are not always appropriate for CPS.

C2. Inter-connectedness leads to complex inter-dependencies which are difficult to identify and reason about. There may be a problem of undesirable emergent behaviour arising at the interfaces between different systems.

C3. Digitalisation has led to a tendency for homogeneity - the same technical solution (software, hardware and middleware) being used across disparate systems. This is one of many factors, alongside legacy systems and unpatched software, that facilitates the migration of worm malware as seen with the rapid spread of the WannaCry ransomware.

C4. The increasing reliance on Artificial Intelligence solutions - particularly Machine Learning - which are difficult to reason about.

In the US, infrastructure security falls within the remit of the Cybersecurity and Infrastructure Security Agency (CISA) ${ }^{5}$ which is part of the Department of Homeland Security. CISA has identified a number of emerging trends that impact on the security of ICS; in addition to some of the challenges identified by RITICS they include:

- Bring Your Own Device (BYOD) - possibly surprising but some vendors have used the possibility of BYOD to access control systems, particularly in an Industrial Internet of Things setting, as a major selling point.

- Virtual Machine Technologies - which is often seen as a way of reducing capital equipment costs by, for example, hosting the Demilitarised Zone and the ICS servers on the same physical machine.

- ICS Supply Chain Management - many suppliers of equipment and software for critical infrastructure source components from around the world. Major systems, such as aircraft, may rely on thousands of suppliers. Managing such an ecosystem and ensuring the security of the end product is becoming a major challenge.

\footnotetext{
${ }^{4}$ This is debatable; for example, whilst a cryptographic algorithm may be insecure, it might require so much computational resource to break that it is still safe to use it - although emerging technologies such as quantum computation may change this argument.

${ }^{5}$ https://www.cisa.gov/national-cybersecurity-communications-integration-center
} 
- Leveraging Cloud Services in ICS - some researchers and organisations are beginning to consider how cloud services may be used for data storage and to provide other services to support their ICS architectures.

\section{Our approach}

We are working in a number of areas which address some of these challenges and we highlight three pieces of work in this section.

\subsection{Real-time CPS Monitoring for Security Research}

Monitoring systems are essential to understand and control the behaviour of systems and networks. CPS are particularly delicate under that perspective since they involve real-time constraints and physical phenomena that are not usually considered in common IT solutions. Unfortunately, the security research community lacks open and configurable monitoring tools that can be adapted to different CPS research scenarios and consider real-time aspects as well. Therefore, there is a need for publicly available monitoring tools able to contemplate these aspects. We have developed an approach, called CPS-MT [1], which is a proof-of-concept of a versatile, real-time CPS monitoring tool, with a particular focus on security research.

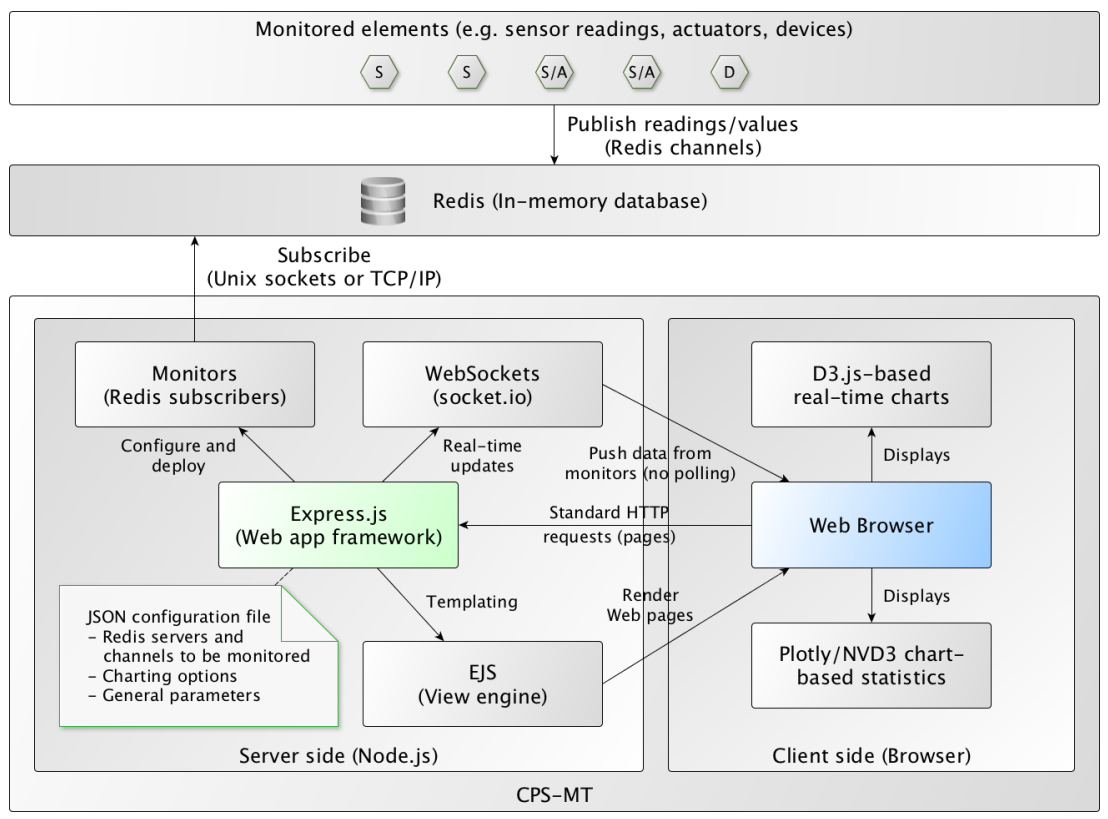

Fig. 2. CPS-MT High Level Architecture 
Our monitoring approach relies on the architecture illustrated in Fig. 2. The upper layer represents the CPS elements to be monitored, e.g., readings from PLCs, actuators, sensor readings, etc. The middle layer acts as a broker between the elements being monitored and CPS-MT. We use Redis [2] to implement this layer. Redis is a fast in-memory database that stores data in the form of keyvalue pairs. The main idea is that monitored elements publish their data via Redis channels and CPS-MT subscribes to these predefined channels in order to receive updates in real time. This makes CPS-MT almost agnostic to what is being monitored, and thus very flexible. Since our main focus is on security research, we assume that cyber attacks conducted within the framework will not target Redis but rather the CPS components within the experimental setup. This is because the role of Redis it to record the data during an attack (e.g. what data PLCs and sensors send, what PLCs receive, etc.) for posterior analysis and research. The bottom layer illustrates the main components of the CPSMT client-server architecture. The main goal of the server is to monitor the activity on Redis channels and report updates to the client side. The client (Web browser) will display and/or capture this new data as it becomes available. We use WebSockets to implement a two-way communication between the server and the client [3]. This allows the server to push data directly into the client in real

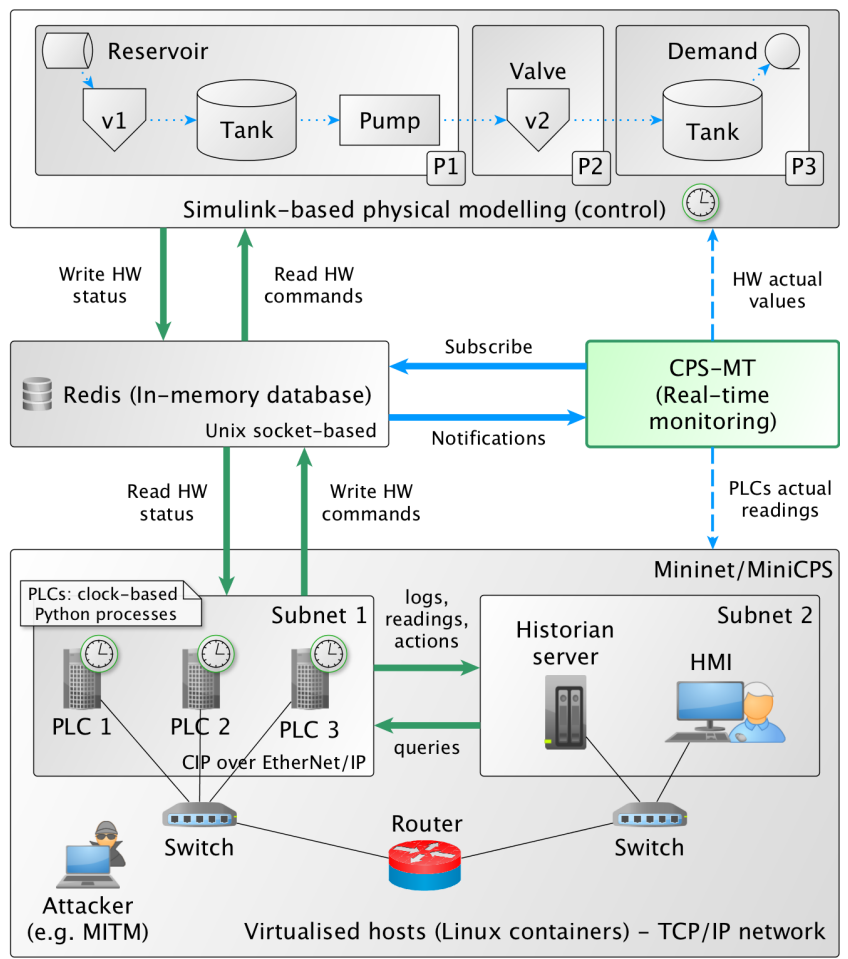

Fig. 3. CPS-MT Deployment 
time. WebSockets eliminate long polling and multiple client requests as happens with traditional HTTP-based approaches. Visualisation aspects are handled by the client side and rely mostly on JavaScript and D3.js [4]. The system also allows the exploration of captured sessions in order to analyse CPS behaviour over specific periods of time.

We have used CPS-MT to analyse the behaviour and impact of MITM attacks over a simulated water treatment plant built with MiniCPS [5]. Fig. 3 shows the details of the CPS-MT deployment within this scenario. MiniCPS is an extensible Python-based simulation framework built on top of Mininet [6], which implements simulated CPS components such as PLCs, their interactions with physical devices, and standard industrial protocols such as Modbus/TCP and CIP over Ethernet/IP. We have extended MiniCPS to also support Redis as its data store, thus enabling CPS-MT to monitor the status of the whole simulation process, including cyber attacks.

\subsection{Measuring Cyber-Physical Security}

Over the last years, ICS have become increasingly exposed to a wide range of cyber-physical threats. Efficient models and techniques able to capture their complex structure and identify critical cyber-physical components are therefore essential. AND/OR graphs have proven very useful in this context as they are able to represent intricate logical interdependencies among ICS components. However, identifying critical nodes in AND/OR graphs is an NP-complete problem. In addition, ICS settings normally involve various cyber and physical security measures that simultaneously protect multiple ICS components in overlapping manners, which makes this problem even harder. For example, a number of sensors and actuators may be protected by a fenced area but some of them may be additionally secured in a locked enclosure. In [7] we have developed a

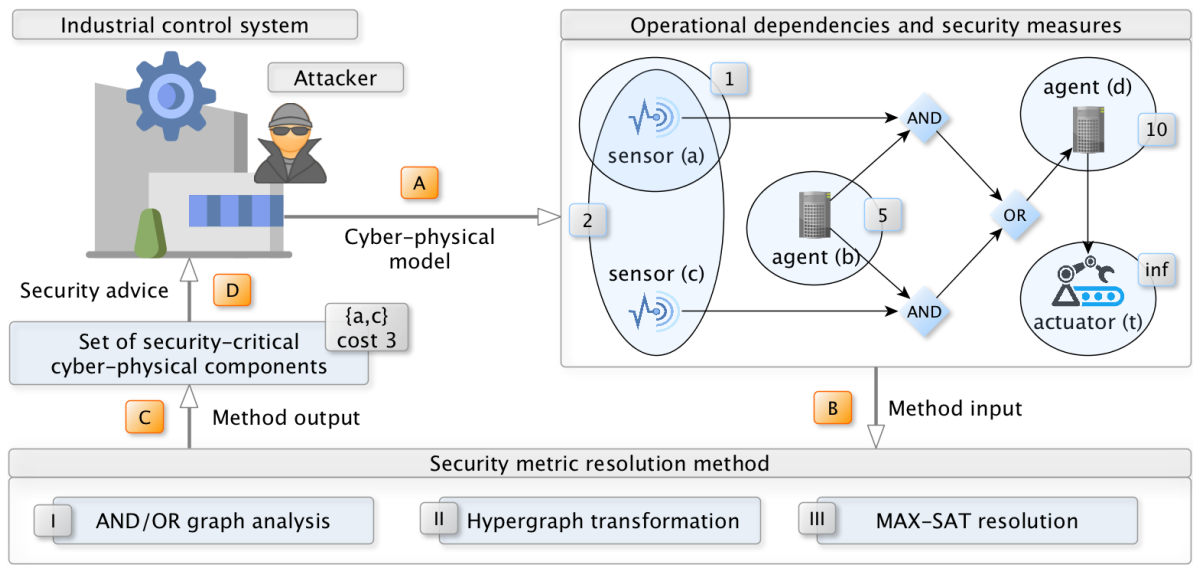

Fig. 4. Measuring ICS cyber-physical security via minimum-effort attack strategies 
security metric based on weighted AND/OR hypergraphs which efficiently identifies the set of critical ICS components and security measures that should be compromised, with minimum cost (effort) for an attacker, in order to disrupt the operation of vital ICS assets. In particular, we use AND/OR hypergraphs to model dependencies between ICS components and the security measures used to protect them as shown in Fig. 4. We then transform this model into a weighted logical formulation that is used to solve a maximum satisfiability (MAX-SAT) problem. The solution to this problem indicates the minimum cost set of cyberphysical components and security measures that must be compromised to disrupt the operation of the system.



Fig. 5. The output from META4ICS for scenario in Fig. 4

We have developed an open source tool called META4ICS [8] that implements this methodology and outputs the computed metric in a JSON file that is later used to display the solution on a web browser. Fig. 5 shows the output of META4ICS for the ICS scenario illustrated in Fig. 4 where the critical components $\{a, c\}$ are highlighted with red dashed circles and the critical security measures $\{s 1, s 3\}$ are underlined and highlighted in red. META4ICS uses a parallel SAT-solving architecture which increases its performance and allows it to scale to graphs with thousands of nodes in seconds. In particular, a significant part of our evaluation benchmark [9] has been included in the body of optimization problems used in the MaxSAT Evaluation $2019^{6}$ to assess the participant MaxSAT solvers. Interestingly, none of the solvers evaluated in the competition performed better than the others on every instance of our dataset. The reason is that distinct MaxSAT solvers generally use very different resolution techniques. As a consequence, the outcome obtained from diversity (using parallel solving) is in fact quite good. Although further work is required, these results also indicate that our approach can be potentially used in dynamic scenarios to prioritise assets and security measures during ongoing intrusions and cyber attacks.

\footnotetext{
${ }^{6}$ https://maxsat-evaluations.github.io/2019/
} 


\subsection{Software Diversity}

It has been recognised since the 1970s that diversity in software can lead to increased reliability in embedded systems. Baudry and MonPerrus [10] provide an excellent survey of different approaches. The survey article distinguishes two main themes in diversity engineering: managed diversity; and automated diversity. A key technique in the former is n-version design or programming. Automated approaches include randomisation techniques and obfuscation.

One can consider diversity at different scales ranging from individual statements within a program through larger code fragments to the level of networks. Within the managed diversity strand, O'Donnell and Sethu [11] consider software diversity at the level of networks, they present the allocation of different software packages to nodes in a network as a graph colouring problem and show how diversity can improve the resilience of a network.

Building on the work of O'Donnell and Sethu, we [12] have developed an approach at the network level. In contrast to much of the earlier work, we model networks in which nodes may be running multiple, vulnerable products and in which there may be constraints on which products can cohabit a node - for example some products will not run on certain operating systems. The main objective of our work is to study the similarities between products which may cause malware to propagate more rapidly through a network - the output is an optimal allocation of products to nodes that slows malware propagation as far as possible.

We use a notion of similarity which is based on shared vulnerabilities; information about vulnerabilities can be extracted from the National Vulnerability Database (NVD) using tools such as CVE-SEARCH [13]. The similarity of two products is computed as the number of shared vulnerabilities divided by the total number of vulnerabilities of the two - this is called the Jaccard similarity coefficient. The Jaccard similarity coefficient is one way to quantify how similar a pair of software products are, giving a measure of how likely it is that they could be affected by the same malware. It is important to note that, even though we use a similarity measure based on shared vulnerabilities, other measures such as the amount of shared code base could be used instead. The underlying premise is that the more similar two products are, whatever the measure, the more likely it is that malware (even 0-days) will propagate between them. Our work allows multiple services, each of which can be realised by multiple products, to be assigned to each node; it also allows local and global constraints on the combination of products in a solution. The optimisation problem can be efficiently solved and results in an assignment of products to nodes.

We then measure the improved resilience of the diversified network in terms of a network diversity metric [14] and Mean-time-to-compromise (MTTC), to verify the effectiveness of our approach. We have shown the competitive scalability of our approach in finding optimal solutions within a couple of seconds to minutes for networks of large scale (up to 10,000 hosts) and high density (up to 240,000 edges). 
Whilst it is true that the NVD concentrates on vulnerabilities in software that is typically deployed on traditional IT systems it is noteworthy that, for example, it contains nearly 200 vulnerabilities that relate to programmable logic controllers (PLCs) and that over $20 \%$ of these have been published since January 2019. It is to be expected that, as cyber physical systems become more prevalent, the rate of discovery of new vulnerabilities in devices and products developed for such systems will rapidly increase.

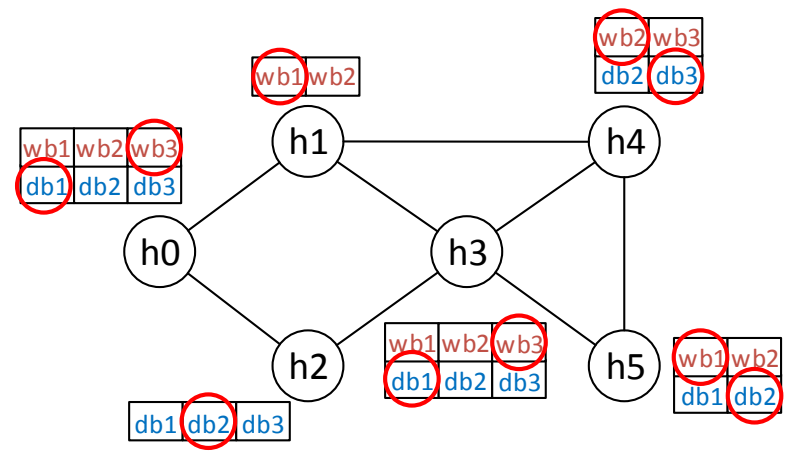

Fig. 6. One possible allocation

A simple example of the output of the work from [12] is shown in Fig 6 - in this simple network, there is a choice between various web browsers and database systems at each node. The allocation is illustrated by the red circles. Currently, our approach optimises the allocation of network resources from a static perspective. However, it could be integrated with vulnerability management tools to support dynamic network reconfiguration as new vulnerabilities and security patches are disclosed. Moreover, the proposed technique could also be used to improve existing defence methods such as Moving Target Defence (MTD) and design diversity-based MTD methods to address dynamic scenarios.

\section{Conclusions}

We have presented our work which addresses some of the challenges identified in the opening sections of this short paper. In each case, the work has been supported by proof-of-concept implementations and, in the cited papers, we have demonstrated that the approaches scale appropriately.

Our work on monitoring provides an experimental framework that could be used to evaluate different approaches to intrusion detection and prevention. It also provides an experimental infrastructure which can be used to help cyber security professionals to gain a better understanding of CPS in line with challenge $\mathrm{C} 1$. In the long term, we envisage a hybrid infrastructure with hardwarein-the-loop and emulations of typical control systems components.

The work on measurement explicitly addresses the inter-dependencies inside a critical infrastructure. This is a first step and does not directly address the 
challenge $\mathrm{C} 2$ about emergent behaviour identified earlier. However, there is no a priori reason why our techniques could not be used to study inter-dependencies in Systems of Systems. This may require an enrichment of the logic used to represent dependencies beyond the simple propositional connectives used at present.

It is well-recognised that software/hardware diversity improves resilience of systems in the face of increasing digitalisation as stated in challenge C3. Our work represents an advance on the state-of-the-art in modelling multidimensional diversity in a network whereas previous work has been based on much simpler graph colouring. There is a cost associated with diversity, both in terms of training and higher operational costs (e.g. licensing, maintenance). We currently optimise for reducing the rate of malware propagation in a network but future work should take these socio-technical considerations into account. This could involve a game-theoretic approach, as used in [15], which can account for various types of cost. Others, for example Michael Franz [16], are actively working on practical and inexpensive approaches to software diversity.

Machine Learning algorithms are increasingly used in security applications for intrusion detection both in enterprise IT systems and, more recently in ICS. It is also now well-known that such algorithms are susceptible to attack by "poisoning" of training data or through techniques involving the introduction of perturbations in live data which are designed to be undetectable. This has led to a sub-discipline of adversarial machine learning. In $[17,18]$ we examine the vulnerability of such intrusion detection systems to adversarial attacks. The attacker is able to manipulate the data sent to an IDS and seeks to hide their presence. This is proving to be a fruitful area of research. Our preliminary results are promising so far, although we plan to further extend our contributions and also consider fundamental aspects aligned with challenge $\mathrm{C} 4$ such as interpretability, transparency, and explainability for AI-based trustworthy cyber-physical systems.

Other partners in RITICS have been studying issues relating to supply chain security (particularly in the context of the EU Network and Information Security (NIS) Directive - see [19] for example), the interactions between various nonfunctional requirements in a CPS (notably safety and security) and the secure use of cloud services. Further details can be found on the RITICS website ${ }^{7}$ and the KIOS Centre of Excellence (KIOS $\mathrm{CoE})^{8}$ which has partially funded and supported our work.

\section{References}

1. Barrère M., Hankin C., Barboni A., Zizzo G., Boem F., Maffeis S. and Parisini T.: CPS-MT: A Real-Time Cyber-Physical System Monitoring Tool for Security Research. 24th IEEE International Conference on Embedded and Real-Time Computing Systems and Applications, pp. 240-241, IEEE Computer Society, 2018. https://doi.org/10.1109/RTCSA.2018.00040

2. Redis. https://redis.io/. Accessed: July 2020.

\footnotetext{
7 https://ritics.org

8 https://www.kios.ucy.ac.cy
} 
3. RFC 6455 - The WebSocket Protocol. https://tools.ietf.org/html/rfc6455. Accessed: July 2020.

4. D3.js - Data Driven Documents. https://d3js.org/. Accessed: July 2020.

5. Antonioli D. and Tippenhauer N. O.: MiniCPS: A Toolkit for Security Research on CPS Networks. In Proceedings of the First ACM Workshop on Cyber-Physical Systems-Security and/or PrivaCy, CPS-SPC'15, 2015.

6. Mininet. http://mininet.org/. Accessed: July 2020.

7. Barrère M., Hankin C., Nicolaou N., Eliades D. G. and ParisiniT.: Measuring cyber-physical security in industrial control systems via minimum-effort attack strategies. Journal of Information Security and Applications 52, (2020). https://doi.org/10.1016/j.jisa.2020.102471

8. Barrère M.: META4ICS - Metric Analyser for Industrial Control Systems, https: //github.com/mbarrere/meta4ics, May 2019.

9. Barrère M., Hankin C., Nicolaou N., Eliades D. G. and ParisiniT.: MaxSAT Evaluation 2019 - Benchmark: Identifying Security-Critical Cyber-Physical Components in Weighted AND/OR Graphs. In MaxSAT Evaluation 2019 (MSE'19), 2019. https://arxiv.org/abs/1911.00516

10. Baudry B. and Monperrus M.: The Multiple Facets of Software Diversity: Recent Developments in Year 2000 and Beyond. ACM Computing Surveys 48(1), 2015.

11. O'Donnell A. J. and Sethu H.: On achieving software diversity for improved network security using distributed coloring algorithms, in Proceedings of the 11th ACM Conference on Computer and Communications Security, pp 121-131, ACM New York, 2004.

12. Li T., Feng C. and Hankin C.: Improving ICS Cyber Resilience through Optimal Diversification of Network Resources. CoRR, abs/1811.00142, 2018. To appear at DSN 2020. http://arxiv.org/abs/1811.00142

13. Moreels P.-J. and Dulaunoy A.: CVE-SEARCH, GitHub repository at https:// github.com/cve-search/cve-search. Accessed: July 2020.

14. Zhang M., Wang L., Jajodia S., Singhal A., and Albanese M.: Network Diversity: A Security Metric for Evaluating the Resilience of Networks Against Zero-Day Attacks. IEEE Transactions on Information Forensics and Security 11(5), pages 1071-1086, 2016.

15. Fielder A., Panaousis E.A., Malacaria P., Hankin C., and Smeraldi F.: Decision support approaches for cyber security investment. Decision Support Systems, 86, pages 13-23, 2016. https://doi.org/10.1016/j.dss.2016.02.012.

16. Franz M.: Making Multivariant Programming Practical and Inexpensive. IEEE Security \& Privacy, 16, pages 90-94, May/June 2018. https://doi.org/10.1109/MSP.2018.2701161

17. Zizzo G., Hankin C., Maffeis S. and Jones K.: Adversarial Machine Learning Beyond the Image Domain. Proceedings of the 56th Annual Design Automation Conference 2019, page 176, ACM Press, 2019. https://doi.org/10.1145/3316781.3323470

18. Zizzo G., Hankin C., Maffeis S. and Jones K.: Intrusion Detection for Industrial Control Systems: Evaluation Analysis and Adversarial Attacks. CoRR, abs/1911.04278, 2019. http://arxiv.org/abs/1911.04278

19. Michalec A., Van Der Linden D., Milyaeva S. and Rashid A.: Industry Responses to the European Directive on Security of Network and Information Systems (NIS): Understanding policy implementation practices across critical infrastructures. https://research-information.bris.ac.uk/en/publications/ industry-responses-to-the-european-directive-on-security-of-netwo. Accessed: July 2020. 